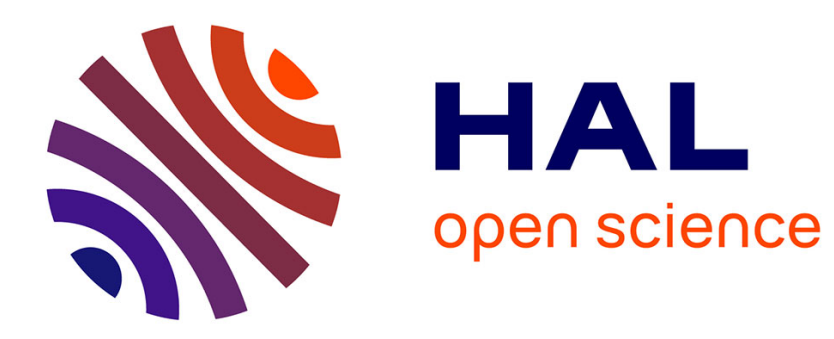

\title{
Random (free) energies in spin glasses
}

Cirano de Dominicis, H.J. Hilhorst

\section{To cite this version:}

Cirano de Dominicis, H.J. Hilhorst. Random (free) energies in spin glasses. Journal de Physique Lettres, 1985, 46 (19), pp.909-914. 10.1051/jphyslet:019850046019090900 . jpa-00232917

\section{HAL Id: jpa-00232917 https://hal.science/jpa-00232917}

Submitted on 1 Jan 1985

HAL is a multi-disciplinary open access archive for the deposit and dissemination of scientific research documents, whether they are published or not. The documents may come from teaching and research institutions in France or abroad, or from public or private research centers.
L'archive ouverte pluridisciplinaire HAL, est destinée au dépôt et à la diffusion de documents scientifiques de niveau recherche, publiés ou non, émanant des établissements d'enseignement et de recherche français ou étrangers, des laboratoires publics ou privés. 
Classification

Physics Abstracts

64.70P $-05.40-75.40$

\title{
Random (free) energies in spin glasses
}

\author{
C. de Dominicis
}

Service de Physique Théorique, CEN Saclay, 91191 Gif sur Yvette Cedex, France

and H. J. Hilhorst

Laboratory of Applied Physics, BP 5046, 2600 GA Delft, The Netherlands

(Reçu le 2 juillet 1985, accepté le 19 août 1985)

Résumé. - Nous étudions le modèle d'énergies aléatoires généralisé de Derrida et la loi de probabilité qui en résulte pour les poids des niveaux d'énergie (libre). Nous montrons que celui-ci peut être utilisé pour représenter les énergies libres (dégénérées) du verre de spin dans la limite de champ moyen. Nous complétons ainsi la représentation proposée récemment par Mézard-Parisi-Virasoro.

\begin{abstract}
We study the Derrida generalized random energy model and its resulting probability law for the weights of the (free) energy levels. We show that it can be used to represent the (degenerate) free energies of the mean field spin glass thus completing the picture proposed by Mézard-Parisi and Virasoro.
\end{abstract}

The Random Energy Model (REM) was introduced by Derrida [1] as a simplification of spin glass problems. The model consists of $\alpha_{1}^{N}$ independent random energy levels $E_{\alpha}$ drawn from a Gaussian probability law $\mathfrak{T}_{1}(E) \sim \exp -\left(E^{2} / N J^{2} a_{1}\right)$ and carrying a weight $P_{\alpha}=\exp -\beta E_{\alpha} / Z$, $Z=\sum_{\alpha} \exp -\beta E_{\alpha^{*}}$. At some critical temperature $T_{1}=\left(a_{1} / 4 \ln \alpha_{1}\right)^{1 / 2}$ the system reaches zero entropy and «freezes ». Recently Derrida and Toulouse [2] were able to directly compute the moments of the probability law for the weights

$$
W^{(k)}=\sum_{\alpha}\left\langle\left(P_{\alpha}\right)^{k}\right\rangle
$$

where $\left\langle>\right.$ stands for average over the Gaussian distributions $\mathfrak{T}_{1}$. They found that the $W^{(k)}$ were the same functions as those computed a while ago [3] (via replicas) for the Sherrington-Kirkpatrick (SK) model [4]. In the REM the argument of the $W$ 's is $T / T_{1}$, in the SK model $x\left(q_{\mathrm{M}}\right)$, the edge of the upper plateau of the Parisi function, a correspondence that had been established by Gross and Mézard [5] starting from a spin representation [1] of the Derrida model.

Trying to take into account correlations between levels, Derrida has introduced recently [6] a Generalized Random Energy Model (GREM) where now the random levels are built as sum 
of $R$ independent random variables

$$
E_{\alpha} \equiv E_{j_{1} j_{2} \ldots j_{R}}=\varepsilon_{j_{1}}+\varepsilon_{j_{1} j_{2}}+\cdots+\varepsilon_{j_{1} j_{2} \ldots j_{R}}
$$

where $j_{r}=1,2, \ldots, \alpha_{r}^{N}$. Each of the $\varepsilon_{j_{1} j_{2} \ldots j_{r}}$ is drawn from a new Gaussian probability law

$$
\mathfrak{T}_{r}(\varepsilon) \sim \exp -\left(\varepsilon^{2} / N J^{2} a_{r}\right) .
$$

The model exhibits $R$ transitions at $T_{r}=\left(a_{r} / 4 \ln \alpha_{r}\right)^{1 / 2}$ (provided the parameters are such that $T_{r}<T_{r-1}$ ). Below $T_{r}$ the tiers 1 through $r$ are "frozen " in the sense that

(i) the thermal averages of $\varepsilon_{j_{1}}, \ldots, \varepsilon_{j_{1} \ldots j_{r}}$ become temperature independent $E_{1}, \ldots, E_{r}$ (with zero associated entropy),

(ii) the « efficacious " levels are no longer exponential in number, but a smaller number in the vicinity of $E_{1}, \ldots, E_{r}$.

The hierarchical tree structure of the levels with $R$ tiers thus provides a natural definition of the (co)distance $r$ (or $x_{\mathrm{r}} \equiv r / R$ ) between two levels whose energies have $r$ identical components $\varepsilon_{j_{1}}, \varepsilon_{j_{1} j_{2}}, \ldots, \varepsilon_{j_{1} j_{2} \ldots j_{r}}$ but a distinct $(r+1)$ th component. Still missing is a spin interpretation that (via a proof à la Gross-Mézard) would provide a direct definition of a monotonic overlap function $q_{r}$ between those two levels.

Here we want to study the moments of the probability law for the weights $P_{j_{1} j_{2} \ldots j_{R}}$ of levels having a given (co)distance $x_{r}$

$$
W_{r}^{(k)}=\sum_{j_{1} \ldots j_{r}}\left\langle\left(\sum_{j_{r+1} \ldots j_{R}} P_{j_{1} \ldots j_{r} j_{r+1} \ldots j_{R}}\right)^{k}\right\rangle .
$$

The first part of this paper provides an explicit form for $W_{r}^{(k)}$ at all temperatures.

Independently of the above developments of the GREM, Mézard, Parisi and Virasoro [7] have proposed a description of the standard mean field (SK) model that illuminates all the properties (e.g. sample to sample or valley to valley fluctuations) discovered in previous studies $[3,8]$. They write the free energy of a state $\alpha$ as

$$
F_{\alpha}=F_{0}+f_{\alpha}
$$

with $F_{0}$ the extensive, degenerate part and $f_{\alpha}$ a fluctuation of relative order $1 / N$ and they assume that $f_{\alpha}$ is an independent random variable with an exponential probability law

$$
Q(f)=\rho_{\mathrm{M}} \exp \left\{\rho_{\mathrm{M}}\left(f-f^{0}\right)\right\} \theta\left(f^{0}-f\right)
$$

with $\rho_{\mathrm{M}}=\beta x\left(q_{\mathrm{M}}\right)$. They then show that all the moments $W^{(k)}$ (Eq. (1)) are identically those of the probability law for the weights $P_{\alpha}$ of reference [3]. Further defining the weight of a cluster [3] $\mathrm{I}_{a}$ characterized by the overlap $q, P_{\mathbf{I}_{q}}=\sum_{\alpha \in \mathbf{I}_{q}} P_{\alpha}$, they show that the same result holds provided $\rho_{\mathrm{M}}$ is replaced by $\rho_{q}=\beta x(q)$.

They, however, do not say how one could define the free energy associated with a cluster and a naive but natural definition such as

$$
\exp -\beta f_{\mathrm{I}}=\sum_{\alpha \in \mathrm{I}} \exp -\beta f_{\alpha}
$$

leads to inconsistencies $\left({ }^{1}\right)$ as we shall see below. This has led us to propose to represent the free

$\left({ }^{1}\right)$ E.g. in dynamics [9] we are interested in computing $\langle\exp -t Z\rangle$. Then depending upon whether we choose to write $Z$ as $\sum_{\alpha}$ or $\sum_{\mathrm{I}_{q}}$ we get $\exp -(t) x\left(q_{\mathrm{M}}\right)$ or $\exp -(t) x(q)$ respectively. 
energies, along the lines of the GREM, as a sum of $R$ components

$$
F_{\alpha} \equiv F_{j_{1} j_{2} \ldots j_{R}}=f_{j_{1}}+f_{j_{1} j_{2}}+\cdots+f_{j_{1} j_{2} \ldots j_{R}}
$$

We show below that, provided we appropriately choose the parameters viz.

$$
T / T_{r}=x\left(q_{r}\right)
$$

we obtain a representation for the free energies of the SK model that reproduces all desired features (moments) and leaves no ambiguity for the definition of the cluster free energy $\left({ }^{2}\right) f^{(r)}$ (with codistance $x_{r}$, or overlap $q_{r}$ ) i.e.

$$
\exp \left(-\beta f_{j_{1} \ldots j_{r}}^{(r)}\right)=\exp \left[-\beta\left(f_{j_{1}}+\cdots+f_{j_{1} \ldots j_{r}}\right)\right] \sum_{j_{r+1} \ldots j_{R}} \exp \left[-\beta\left(f_{j_{1} \ldots j_{r+1}}+\cdots+f_{j_{1} \ldots j_{R}}\right)\right]
$$

This new proposal is the content of the last part of this paper.

1. On the moments $W_{r}^{(k)}$.

In the GREM the result for equation (4) reads

$$
\begin{aligned}
W_{r}^{(k)} & =\Gamma\left(k-T / T_{r}\right) / \Gamma(k) \Gamma\left(1-T / T_{r}\right) & & T<T_{r} \\
& =0 & & T>T_{r}
\end{aligned}
$$

in agreement with the result of Derrida-Toulouse for $r=1$. Starting from the paramagnet $\left(T>T_{1}\right)$ and cooling down, one sees at $T_{1}$ the first tier freezing, i.e. $\widetilde{\varepsilon}_{j_{1}}=E_{1}+\varepsilon_{j_{1}}$ and the $\varepsilon_{j_{1}}$ confined near $E_{1}$. Correspondingly $W_{1}^{(k)}$ starts being non zero (some levels $\varepsilon_{j_{1}}$ start to dominate). At $T_{2}$ the same phenomenon occurs with the second tier etc. Below $T$ all $W^{(k)}$ are non-zero and " all tiers are frozen ".

As mentioned above there are two aspects in freezing :

(i) Frozen (free) energies $E_{r}$

$$
E_{r}=-N\left(a_{r} \ln \alpha_{r}\right)^{1 / 2}+\left(a_{r} / 16 \ln \alpha_{r}\right)^{1 / 2} \ln N
$$

whose sum over $r$ is the (free) energy $E$ of the system.

(ii) Averages over $\alpha_{r}^{N}$ levels drawn from $\mathfrak{T}_{r}(\varepsilon)$ (Eq. (3)) may be replaced by averages on $M_{r}$ lowest levels, all located near $E_{r}$, the « efficacious levels », with a distribution (cf. Eq. (6))

$$
\mathfrak{T}_{r}(\widetilde{\varepsilon})=\left(1 / T_{r}\right) \exp \left\{\left(\widetilde{\varepsilon}-\varepsilon_{r}^{0}\right) / T_{r}\right\} \theta\left(\varepsilon_{r}^{0}-\widetilde{\varepsilon}\right)
$$

together with, for $M_{r} \rightarrow \infty$

$$
M_{r} \exp -\left(\varepsilon_{r}^{0} / T_{r}\right)=\left(4 \pi \ln \alpha_{r}\right)^{-1 / 2} \equiv V_{r} .
$$

This result is implicit in Mézard-Parisi-Virasoro and is derived by replacing $\varepsilon$ by $E_{r}+\widetilde{\varepsilon}$ in (3), using (12),

$$
M_{r} \sim\left(\alpha_{r}\right)^{N} \int_{-\infty}^{E_{r}+\varepsilon_{r}^{0}} T_{r}(\varepsilon) \mathrm{d} \varepsilon
$$

and expanding for large $N$.

$\left({ }^{2}\right)$ Neither for the evaluation [10] of $\langle\exp -t Z\rangle$. 


\section{Sketch of derivation, $R=2$.}

The tired reader may jump to section 3 . We compute $W_{2}^{(2)} \equiv \sum_{j_{1} j_{2}}\left\langle P_{j_{1} j_{2}}^{2}\right\rangle$

$$
\begin{aligned}
W_{2}^{(2)}=\left(\alpha_{1} \alpha_{2}\right)^{N} \int_{0}^{\infty} \mathrm{d} \lambda \lambda\left\{\left[\int \mathrm{d} \varepsilon_{1} \mathfrak{T}_{1}\left(\varepsilon_{1}\right) f_{0}^{\alpha_{2}^{N}}\left(\mu_{1}\right)\right]^{\alpha_{1}^{N}-1} \times\right. \\
\left.\times \int \mathrm{d} \varepsilon_{1} \mathfrak{T}_{1}\left(\varepsilon_{1}\right) \mathrm{e}^{-2 \beta \varepsilon_{1}} f_{0}^{\alpha_{2}^{N}-1}\left(\mu_{1}\right) f_{2}\left(\mu_{1}\right)\right\}
\end{aligned}
$$

$f_{k}\left(\mu_{1}\right)=\int \mathrm{d} \varepsilon_{2} \mathfrak{T}_{2}\left(\varepsilon_{2}\right) \exp -\left(\mu_{1} \mathrm{e}^{-\beta \varepsilon_{2}}\right) \mathrm{e}^{-k \beta \varepsilon_{2}}$

$\mu_{1}=\lambda \mathrm{e}^{-\beta \varepsilon_{1}}$

(i) $T<T_{2}<T_{1}: \mathscr{T}_{r}(r=1,2)$ become exponential distributions $\tilde{\mathfrak{T}}_{r}$ and $\alpha_{r}^{N} \rightarrow M_{r}$ as in equations (13) to (15). We get

$$
f_{2}\left(\mu_{1}\right) \sim\left(T / T_{r}\right)\left(\mu_{1}\right)^{-2+\left(T / T_{r}\right)} \Gamma\left(2-T / T_{r}\right)
$$

also after partial integration

$$
f_{0}^{\alpha_{2}^{N}}\left(\mu_{1}\right) \sim \exp \left\{-v_{r}\left(\mu_{1}\right)^{T / T_{r}} \Gamma\left(1-T / T_{r}\right)\right\} .
$$

Those steps are identical to those taken in Mézard-Parisi-Virasoro. Except that here they are to be iterated. This is easy since the functions keep the same structure yielding

$$
W_{2}^{(2)}=1-T / T_{2} .
$$

(ii) $T_{2}<T<T_{1}$ : The unfrozen tier $r=2$ keeps now the full Gaussian distribution,

$$
f_{k}\left(\mu_{1}\right)=\pi^{-1 / 2} \int_{-\infty}^{+\infty} \mathrm{d} y \mathrm{e}^{-y^{2}} \exp -\left\{\mu_{1} \mathrm{e}^{-A y}\right\} \mathrm{e}^{-k A y}
$$

with $\Lambda^{2}=N \beta^{2} J^{2} a_{2}$. The large $N$ expansion for $f_{k}$ is given in reference [1]. But since $\mu_{1}=\lambda \exp -\beta \varepsilon_{1}$, contains variables to be integrated upon, it is a priori not clear which is the important range of $\mu_{1}$ as $N \rightarrow \infty$. We get

$$
f_{0}^{\alpha N}\left(\mu_{1}\right)=\left[1-\mu_{1} \mathrm{e}^{\Lambda^{2} / 4}+\cdots\right]^{\alpha_{2}^{N}} \sim \exp -\left\{\mu_{1} x_{2}^{N}\right\}
$$

and $x_{2}^{N}=\alpha_{2}^{N} \exp \left(\Lambda^{2} / 4\right)$, the exponentiation in (23) being valid when $\mu_{1} x_{2}^{N} \simeq \mathcal{O}\left(b^{N}\right), b \leqslant 1$.

For $f_{2}\left(\mu_{1}\right)$ depending on the domain we get

$$
\begin{array}{ccc}
\mathrm{e}^{\Lambda^{2}} & \text { if } & \ln \mu_{1}<-\Lambda^{2} \\
{\left[x_{2}^{N} \mu_{1}\right]^{-2-k(T)}\left[x_{2}^{N}\right]^{-k(T) / 2}} & \text { if } & -\Lambda^{2}<\ln \mu_{1}<-\frac{1}{2} \Lambda^{2}
\end{array}
$$

here a temperature dependent factor is omitted and $k(T)=-(1 / 2)\left(1+\left(T / T_{r}\right)^{2}\right)$.

For the $\varepsilon_{1}$ integration again $\alpha_{1}^{N} \rightarrow M_{1}, \mathfrak{T}_{1} \rightarrow \widetilde{\mathfrak{T}}_{1}$ in (16). With any of the domains quoted above the integrals now reduce to types that already occurred in (i). The final $\lambda$ integral is then seen to pick up its main contribution from $\lambda \sim \mathcal{O}\left(x_{2}^{-N} \exp \beta E_{1}\right)$ so that the condition to obtain (23) is 
satisfied with $b=1$ and consistency is assured. The result for $T_{2}<T<T_{1}$ is then

$$
W_{2}^{(2)}=0 \text {. }
$$

(iii) $T_{2}<T_{1}<T$ : In the paramagnetic region Gaussians have to be kept for both tiers, leading again to $W_{2}^{(2)}=0$ but with a distinct approach to zero.

For arbitrary $R$ a hierarchy of integrations to be carried in succession is involved. This complexity is compounded by the fact that, e.g. in $W_{1}^{(2)}=\sum_{j_{1}}\left(\sum_{j_{2}} P_{j_{1} j_{2}}\right)\left(\sum_{j_{2}} P_{j_{1} j_{2}}\right)$ one has to distinguish the case where $j_{2}=j_{2}^{\prime}$ which for $W_{r}^{(k)}$ leads to a proliferation of distinct sectors.

\section{On the definition of the cluster free energy.}

Looking back on to the cluster free energy as defined in (7) together with probability law $Q\left(f_{\alpha}\right)$ (Eq. (6)) one may compute the corresponding law for $f_{\mathrm{I}}$. In the limit $\left(f_{\mathrm{I}}-f^{0}\right) \rightarrow-\infty$ one obtains indeed an exponential distribution but with a decay constant $x\left(q_{\mathrm{M}}\right)$ identical to that of $f_{\alpha}$ (instead of $x(q)$ as it would be naively expected). In contradistinction if we take a GREM with $R=2$

$$
E_{j_{1} j_{2}}=\varepsilon_{j_{1}}+\varepsilon_{j_{1} j_{2}}
$$

and its effective (free) energy $\eta_{j_{1}}$

$$
\exp -\beta\left(\varepsilon_{j_{1}}+\eta_{j_{1}}\right)=\exp -\beta \varepsilon_{j_{1}} \sum_{j_{2}} \exp -\beta \varepsilon_{j_{1} j_{2}}
$$

one finds that the $\eta$ distribution is asymptotically exponential with a decay constant $1 / T_{2}$ as above but the $(\varepsilon+\eta)$ distribution has the smaller decay constant $1 / T_{1}$ (for $\left.T_{2}<T_{1}\right)$ as we expected above.

\section{The SK model as a GREM.}

It is clear now that we may choose to represent the random free energies of the SK model (Eq. (5)) as a sum of independent random free energies (Eq. (8)). Provided we properly choose the parameters $a_{r}, \alpha_{r}$. It suffices for that to recover

(i) the decay constant $x\left(q_{r}\right)$ via

$$
T\left(4 \ln \alpha_{r} / a_{r}\right)^{1 / 2} \equiv T / T_{r}=x\left(q_{r}\right) .
$$

This insures that one has the proper probability law for all $q$ 's derived by Mézard et al. [3].

(ii) the " frozen " values for the (extensive part of the) free energy

$$
-N J\left(a_{r} \ln \alpha_{r}\right)^{1 / 2} \equiv F_{r}=F_{r}^{\mathrm{SK}} \text {. }
$$

The SK free energy for the tier $r$ may be conveniently written using Sompolinsky's representations $[11,12]$ in the Parisi gauge. Some degree of arbitrariness remains since only $\sum_{r} F_{r}^{\mathrm{SK}}=F^{\mathrm{sK}}$ is uniquely defined. Note that $F_{r}^{\mathrm{SK}} \sim \dot{q}_{r}$ hence $F_{r}^{\mathrm{SK}}=0$ on the plateau of $q_{r}$.

Note finally that in the SK model all $W_{r}^{(k)}$ as given in (11) are non zero, and hence from this point of view all tiers are frozen, and all Gaussian become exponentials near their frozen values $E_{r}$. This expect on the plateau of $q_{r}$ where the width of the Gaussian vanishes $\left(a_{r} \sim \dot{q}_{r}\right)$ and the corresponding $f_{j_{1} \ldots j_{r}}$ components disappear. As $T \rightarrow T_{\mathrm{c}}$ all components disappear to only leave the paramagnetic state. 


\section{Acknowledgments.}

This work was performed while one of the authors $(\mathrm{HJH})$ was at the Ecole Nationale Supérieure des Mines de Paris. He also thanks the members of the Groupe de Physique du Solide de l'Ecole Normale Supérieure and of the Service de Physique Théorique du CEN Saclay for hospitality and fruitful interaction. We both thank B. Derrida for several stimulating discussions. One of us (C.D.) also wishes to thank M. Mézard for useful exchanges.

\section{References}

[1] Derrida, B., Phys. Rev. Lett. 45 (1980) 79; Phys. Rev. B 24 (1981) 2613.

[2] Derrida, B., Toulouse, G., J. Physique Lett. 46 (1985) L-223.

[3] Mézard, M., Parisi, G., Sourlas, N., Toulouse, G., Virasoro, M., Phys. Rev. Lett. 52 (1984) 1156.

[4] Sherrington, M., KirKPatrick, S., Phys. Rev. Lett. 32 (1975) 1792.

[5] Gross, D., MÉzard, M., Nucl. Phys. B 240 [FS12] (1984) 431.

[6] Derrida, B., J. Physique Lett. 46 (1985) L-401.

DERRIDA, B., GARDNER, E., preprint 1985.

[7] Mézard, M., Parisi, G., Virasoro, M., J. Physique Lett. 46 (1985) L-217.

[8] Young, A. P., Bray, A., Moore, M., J. Phys. C 17 (1984) L149.

[9] De Dominicis, C., Orland, H., Lainée, F., J. Physique Lett. 46 (1985) L-463.

[10] De Dominicis, C., ICM 1985 preprint.

[11] Sompolinsky, H., Phys. Rev. Lett. 47 (1981) 935.

[12] De Dominicis, C., Gabay, M., Orland, H., J. Physique Lett. 42 (1981) L-523. 